\title{
Síndrome de Cushing Ectópica: Um Desafio Diagnóstico e Terapêutico
}

\section{Ectopic Cushing Syndrome: A Diagnostic and Therapeutic Challenge}

Vera Fernandes ${ }^{1,2,3}$, Ana Margarida Monteiro ${ }^{1}$, Maria Joana Santos Maria Lopes Pereira1 ${ }^{1}$ Olinda Marques $^{1}$

\section{RESUMO}

INTRODUÇÃO: A síndrome de Cushing ectópica é uma entidade rara e a identificação do tumor produtor de hormona adrenocorticotrópica (ACTH) pode ocorrer anos após o diagnóstico da síndrome de Cushing, dificultando a orientação terapêutica destes doentes.

CASO CLíNICO: Mulher de 78 anos referenciada à consulta de Endocrinologia por síndrome de Cushing. Apresentava sinais e sintomas de hipercortisolismo e a investigação demonstrou síndrome de Cushing ACTH dependente, com ressonância magnética hipofisária sem alterações, cateterismo dos seios petrosos negativo e nódulo pulmonar de 8 mm. A cintigrafia com recetores da somatostatina, a PET-68Ga-SRP, a broncofibroscopia e a citologia do aspirado brônquico não revelaram alterações. Por franco agravamento clínico, iniciou-se terapêutica com metirapona, com resposta clínica e analítica muito favorável.

DISCUSSÃO: Este caso revelou-se um desafio diagnóstico e terapêutico. Embora sem localização do tumor primário, atingiu-se o principal objetivo, o controlo do hipercortisolismo, com melhoria da qualidade de vida da doente.

PALAVRAS-CHAVE: Síndrome de ACTH Ectópica/diagnóstico; Síndrome de ACTH Ectópica/tratamento; Síndrome de Cushing/diagnóstico; Síndrome de Cushing/tratamento 


\section{ABSTRACT}

INTRODUCTION: Ectopic Cushing syndrome is a rare disease and the identification of the adrenocorticotropic hormone (ACTH) producing tumour may occur years after Cushing syndrome diagnosis, hampering the therapeutic management of these patients.

CASE REPORT: A 78-year-old woman was referred to the Endocrinology department due to Cushing syndrome. She had signs and symptoms of hypercortisolism and the clinical investigation showed a dependent ACTH Cushing syndrome with a normal pituitary magnetic resonance imaging, negative catheterization of the petrosal sinuses and an 8 mm pulmonary nodule. Scintigraphy with somatostatin receptors, PET-68Ga-SRP, (bronchofibroscopy) and bronchial aspirate cytology were normal. Due to clinical worsening, she started on metyrapone with a very favourable clinical and analytical response.

DISCUSSION: This clinical case was a diagnostic and therapeutic challenge. Although the location of the primary tumour was not found, control of hypercortisolism was achieved, improving the patient's quality of life.

KEYWORDS: ACTH Syndrome, Ectopic/diagnosis; ACTH Syndrome, Ectopic/therapy; Cushing Syndrome/diagnosis; Cushing Syndrome/therapy

\section{INTRODUÇÃO}

A síndrome de Cushing ectópica (SCE) é uma entidade rara, correspondendo a 5\% a 10\% dos casos de síndrome de Cushing (SC) dependente da secreção da hormona adrenocorticotrópica (ACTH). ${ }^{1,2}$ A maioria dos casos de SCE descritos é causada por tumores neuroendócrinos, destacando-se os carcinomas pulmonares de pequenas células, os tumores carcinoides, o carcinoma medular da tiroide, os tumores dos ilhéus pancreáticos e os feocromocitomas. ${ }^{2}$

É mais prevalente no sexo masculino e na faixa etária dos 40-60 anos, mas pode surgir em qualquer idade. ${ }^{3} \mathrm{~A}$ apresentação clínica pode ser muito variável, com alterações psiquiátricas, ortopédicas, intercorrências infeciosas e queixas sistémicas inespecíficas, como astenia ou irritabilidade. ${ }^{1}$ A suspeita clínica é fundamental para o diagnóstico, podendo este demorar meses a anos. Por outro lado, mesmo após o diagnóstico da SCE, a localização do tumor produtor de ACTH pode ocorrer anos após o diagnóstico, dificultando a orientação terapêutica destes doentes. Se a localização do tumor primário não for possível com os meios de diagnóstico atualmente disponíveis, nomeadamente pela pequena dimensão do tumor, o controlo do hipercortisolismo passa a ser o objetivo terapêutico primordial. Este pode ser alcançado recorrendo a adrenalectomia bilateral ou a terapêutica médica com inibidores da esteroidogénese. ${ }^{4}$

Apesar de rara, a SCE associa-se a uma significativa morbimortalidade. A suspeita clínica é fundamental para um diagnóstico precoce e tratamento adequado, que podem ter uma importante repercussão na qualidade de vida do doente.

\section{CASO CLÍNICO}

Mulher de 78 anos, caucasiana, previamente autónoma nas atividades de vida diária e com antecedentes de hipertensão arterial e dislipidemia, referenciada à consulta externa de Endocrinologia por hipercortisolismo.

Apresentava queixas de astenia e edemas dos membros inferiores com cerca de 1 ano de evolução, agravamento da hipertensão arterial, alopecia, polifagia, fraqueza muscular de predomínio proximal com impossibilidade de marcha sem apoio desde há 6 meses e aumento ponderal de 5 kg no último mês. Estava medicada com alprazolam $1 \mathrm{mg}$ bid, clopidogrel 75 mg id, perindopril $4 \mathrm{mg}$ id, indapamida 1,5 mg id e sinvastatina 20 mg id. Após observação prévia por outro Endocrinologista, estava também medicada com cetoconazol 200 mg/dia (bloqueador das enzimas da esteroidogénese dependentes do citocromo P450) desde há cerca de 1 mês, com meIhoria clínica pouco significativa.

Ao exame físico destacava-se aspeto debilitado, alopecia, pletora facial e face em lua cheia, equimoses dispersas, adiposidade cervical posterior (pescoço de búfalo), estrias violáceas inferiores a $1 \mathrm{~cm}$ de diâmetro na região trocantérica e atrofia muscular proximal nos 4 membros.

O estudo analítico revelou perda do ritmo circadiano do cortisol e níveis de ACTH elevados, confirmando a presença de SC ACTH-dependente. Para estudo da causa da síndrome realizou prova com alta dose de dexametasona (2 mg 6/6 horas, durante 48 horas) que revelou ausência de supressão nos níveis de cortisol urinário, e prova de $\mathrm{CRH}$ (corticotropin-releasing hormone) que foi negativa (sem resposta significativa nos níveis de ACTH e cortisol), resultados a favor de uma origem ectópica 
para a secreção de ACTH. A ressonância magnética hipofisária não revelou alterações, pelo que se prosseguiu a investigação com o cateterismo dos seios petrosos. $\bigcirc$ gradiente central-periférico de $\mathrm{ACTH}$ mais elevado foi 1,04 no estádio basal e 1,57 após estimulação com $\mathrm{CRH}$, sendo compatível com produção de ACTH não hipofisária. Neste seguimento foi feita uma tomografia computorizada cervico-toraco-abdominal que revelou um nódulo de $8 \mathrm{~mm}$, localizado no lobo médio do pulmão direito (Fig. 1). Os doseamentos dos marcadores tumorais, calcitonina e cromogranina, foram negativos. Foi submetida, ainda, a cintigrafia com recetores da somatostatina e a cintigrafia com gálio/tomografia emissora de positrões (PET)-68Ga-SRP que não revelaram focos

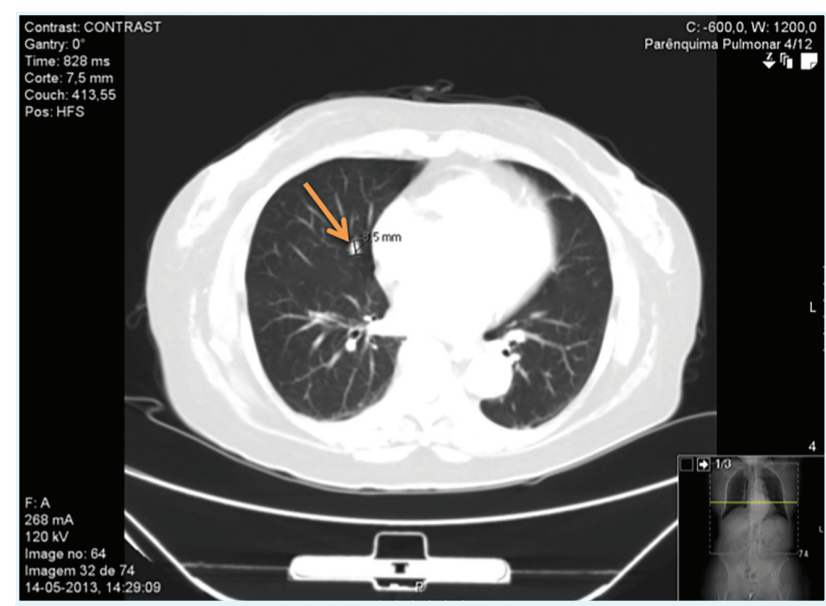

FIGURA 1. Tomografia computorizada cervico-toraco-abdominal nódulo com cerca de $8 \mathrm{~mm}$ localizado no lobo médio do pulmão direito. de hipercaptação. Realizou broncofibroscopia que não revelou alterações e a citologia do aspirado brônquico foi negativa para células neoplásicas. Foi tentada a realização de biópsia pulmonar transtorácica, que não foi possível por formação de hematoma.

Oito meses após a avaliação inicial, a doente foi internada por franco agravamento clínico. A terapêutica com cetoconazol estava suspensa há cerca de 1 mês, por ter sido retirado do mercado. A doente apresentava polidipsia, astenia, dispneia, e não caminhava de forma autónoma. Ao exame físico destacava-se anasarca. Analiticamente, verificava-se hipocaliemia grave (1,7 mmol/L) com tradução eletrocardiográfica e hiperglicemia compatível com diabetes mellitus inaugural. Iniciou reposição endovenosa de potássio e tratamento com doses máximas de espironolactona mas, por ausência de melhoria analítica, decidiu iniciar-se terapêutica médica para bloqueio da síntese de cortisol com metirapona, com titulação de dose até aos $2 \mathrm{~g} /$ dia. Houve uma resposta clínica muito favorável, com melhoria da astenia, do aspeto cushingoide, da fragilidade cutânea e edemas, e com normalização do potássio sérico, melhoria do controlo glicémico e tensional. Constatou-se uma franca diminuição dos níveis de cortisol sérico (80,8\%) e cortisolúria (84,7\%) após cerca de um mês de terapêutica (Fig. 2). A hiperglicemia foi controlada com insulinoterapia. A doente foi observada por Cirurgia Cardiotorácica, que decidiu manter terapêutica conservadora, dado não haver confirmação da origem pulmonar para a produção ectópica de ACTH.

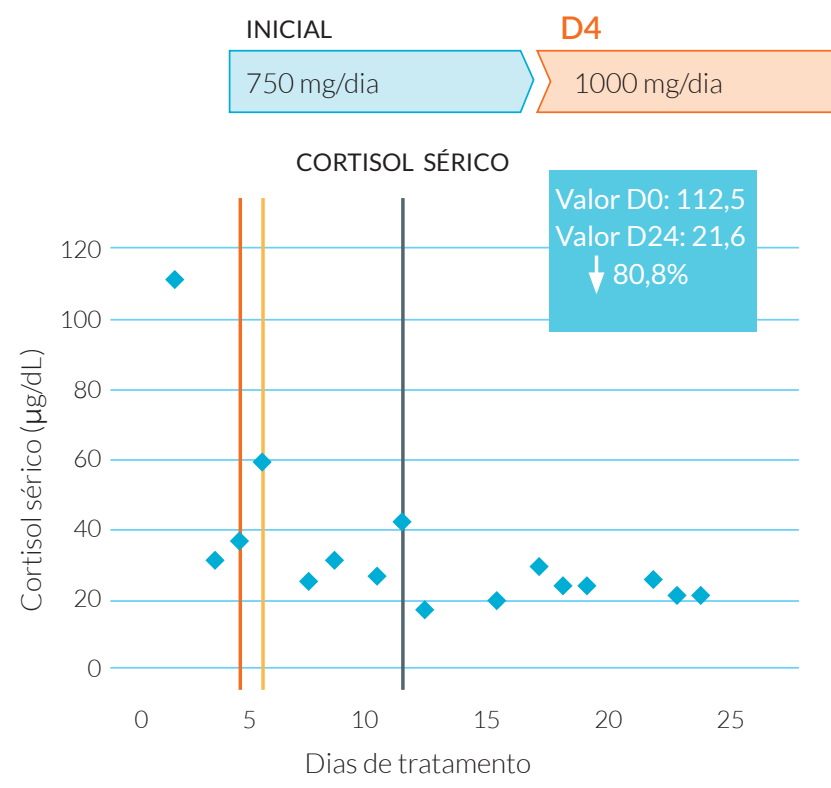

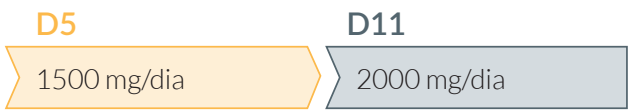

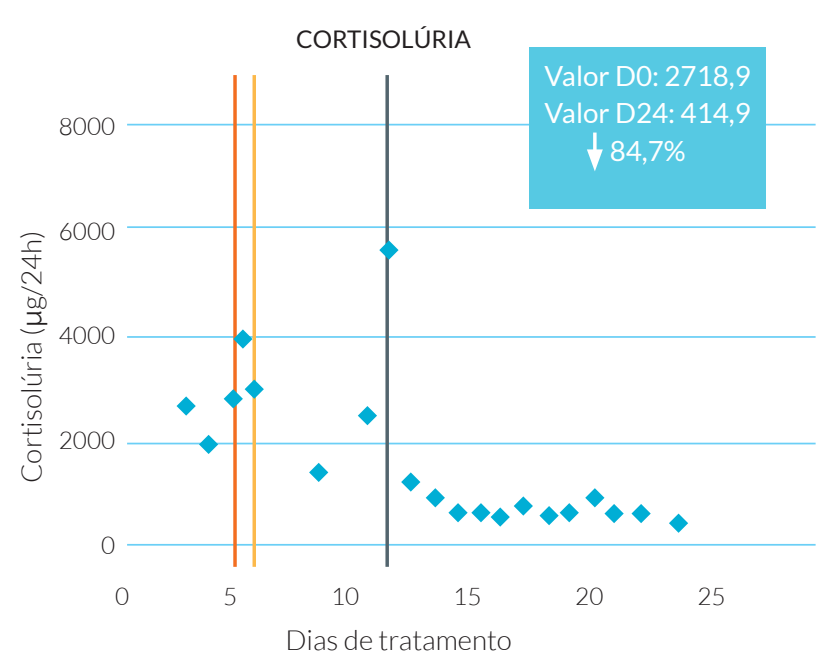

D0, D4, D5, D11, D24: dia 0,4,5,11 e 24, respetivamente, após início de terapêutica com metirapona.

FIGURA 2. Evolução dos níveis de cortisol sérico e cortisolúria após início de metirapona. 
Um mês após o início do tratamento com metirapona, a doente recorreu duas vezes ao Serviço de Urgência por intercorrências infeciosas (urinária e respiratória). Foi constatado um quadro de insuficiência adrenal, tendo sido-instituída terapêutica de substituição com hidrocortisona $30 \mathrm{mg} / \mathrm{dia}$.

Cerca de 30 meses após início de metirapona, mantém terapêutica com metirapona e hidrocortisona. A lesão pulmonar permanece imagiologicamente estável e a evolução clínica tem sido muito favorável, com resolução dos sintomas, recuperação da autonomia da marcha e estabilidade clínica.

\section{DISCUSSÃO}

A doente apresentada teve uma apresentação clínica típica da SCE, de acordo com Ilias I et al, numa série de 90 casos com SCE. Os sinais e sintomas corresponderam aos mais frequentemente descritos nessa série, nomeadamente fraqueza muscular (82\%), hipertensão (78\%), aumento do peso corporal (70\%), hipocaliemia (71\%) e distúrbios psiquiátricos (53\%). ${ }^{1} \bigcirc$ atraso entre o início da sintomatologia e o diagnóstico (1 ano) é também o cenário mais frequentemente encontrado nestes doentes. ${ }^{1}$ Outras fontes referem uma prevalência de hipocaliemia de $80 \% .^{2}$ A hipocaliemia é típica da SCE, em oposição à doença de Cushing, porque o hipercortisolismo é frequentemente severo. Níveis elevados de cortisol impossibilitam a metabolização completa deste em cortisona, a sua forma inativa, pela enzima renal 113 hidroxisteroide desidrogenase tipo 2, permitindo que o cortisol se ligue ao recetor mineralocorticoide no nefrónio distal, promovendo a reabsorção de sódio e a secreção de potássio e, consequentemente, levando a hipertensão e hipocaliemia. ${ }^{4}$

Na investigação etiológica, não se detetou uma fonte inequívoca para a produção de ACTH. A presença do nódulo pulmonar, de dimensões pequenas e estáveis, apoia a hipótese de que a etiologia se deve a um tumor carcinoide. Porém, nenhum dos exames efetuados, ao diagnóstico e no decorrer do seguimento clínico, permitiram ainda confirmar a suspeita. ${ }^{5}$ Acreditamos que tal poder-se-á dever às reduzidas dimensões do tumor e ao seu crescimento indolente. Na série de Ilias I et al, 17 casos (19\%) permaneceram com tumor oculto, com um tempo mediano de seguimento de 26 meses. ${ }^{1}$

O tratamento ideal do SCE passa pela remoção do tumor produtor de ACTH com cura da doença. ${ }^{1,2}$ Como não se conseguiu identificar a origem da produção ectópica de ACTH e, dada a idade e comorbilidades da doente, optou-se pelo tratamento médico para controlo do hiper- cortisolismo. ${ }^{6}$ A adrenalectomia bilateral é também uma possibilidade terapêutica para controlo do hipercortisolismo, sendo utilizada sobretudo quando os inibidores da esteroidogénese são ineficazes ou não tolerados pelo doente. ${ }^{1,4}$ No nosso caso, o tratamento com metirapona foi bem tolerado e revelou-se extremamente eficaz com franca melhoria clínica e analítica após a sua instituição. Alguns destes tumores expressam recetores da somatostatina e foram já descritas terapêuticas dirigidas a estes recetores para o controlo do hipercortisolismo., Neste caso, os análogos da somatostatina não foram utilizados, dado a pesquisa dos referidos recetores ter sido negativa e os bons resultados obtidos com a metirapona.

O prognóstico desta doente é uma incógnita, uma vez que o tumor primário, principal determinante do prognóstico, não foi removido. Porém, estudos prévios descrevem que doentes com SCE proveniente de tumor oculto sobreviveram mais, comparativamente àqueles com tumor identificado. ${ }^{1}$ Por outro lado, nos doentes com tumores identificados, os doentes com tumores pulmonares apresentaram maiores taxas de sobrevivência, exceto nos carcinomas de pequenas-células. ${ }^{1}$ Assim, pensamos que o prognóstico da nossa doente poderá ser mais favorável comparativamente a outras formas de SCE.

Este caso revelou-se um verdadeiro desafio diagnóstico e terapêutico. Embora não tenha sido localizado o tumor primário, atendendo à idade e comorbilidades da doente, consideramos ter sido atingido o principal objetivo (controlo do hipercortisolismo) com melhoria clínica e da qualidade de vida da doente.

\section{AGRADECIMENTOS/ ACKNOWLEDGMENTS}

Os autores gostariam de agradecer à Dra. Selma Souto e à Dra. Marta Alves, assistentes do Serviço de Endocrinologia do Hospital de Braga, pelo contributo de ambas no estudo do presente caso clínico.

CONFLITOS DE INTERESSE: Os autores declaram não ter qualquer conflito de interesse na realização do presente trabalho.

FONTES DE FINANCIAMENTO: Não houve qualquer fonte de financiamento na realização do presente trabalho.

CONFIDENCIALIDADE DOS DADOS: Os autores declaram ter seguido os protocolos da sua instituição acerca da publicação dos dados de doentes.

PROTEÇÃO DE PESSOAS E ANIMAIS: Os autores declaram que os procedimentos seguidos na elaboração do presente trabalho estão em conformidade com as nor- 
mas das comissões de investigação clínica e de ética, bem como da declaração de Helsínquia e da Associação Médica Mundial.

CONFLICTS OF INTEREST: The authors declare that they have no conflicts of interest.

FINANCIAL SUPPORT: This work has not received any contribution, grant or scholarship.

CONFIDENTIALITY OF DATA: The authors declare that they have followed the protocols of their work center on the publication of data from patients.

PROTECTION OF HUMAN AND ANIMAL SUBJECTS: The authors declare that the procedures followed were in accordance with the regulations of the relevant clinical research ethics committee and with those of the Code of Ethics of the World Medical Association (Declaration of Helsinki).

\section{REFERENNCIAS}

1. Ilias I, Torpy DJ, Pacak K, Mullen N, Wesley RA, Nieman LK. Cushing's syndrome due to ectopic corticotropin secretion: twenty years' experience at the National Institutes of Health. J Clin Endocrinol Metab. 2005;90:4955-62.

2. Salgado LR, Fragoso MC, Knoepfelmacher M, Machado MC, Domenice S, Pereira MA, et al. Ectopic ACTH syndrome: our experience with 25 cases. Eur J Endocrinol. 2006;155:725-33.

3. Carroll TB, Aron DC, Findling JW, Tyrrell JB. Glucocorticoids \& Adrenal Androgens. In: Gardner DG, Shoback D, editors. Greenspan's Basic \& Clinical Endocrinology. New York: The McGraw-Hill; 2011. p. 285-327.

4. Nieman LK, Biller BM, Findling JW, Murad MH, Newell-Price J, Savage MO, et al. Treatment of Cushing's syndrome: an Endocrine Society Clinical Practice Guideline. J Clin Endocrinol Metab. 2015;100:2807-31.

5. Nieman LK, Biller BM, Findling JW, Newell-Price J, Savage MO, Stewart PM, et al. The diagnosis of Cushing's syndrome: an Endocrine Society Clinical Practice Guideline. J Clin Endocrinol Metab. 2008;93:1526-40.

6. Miller JW, Crapo L. The medical treatment of Cushing's syndrome. Endocr Rev. 1993;4:443-58.

7. Bruin C, Feelders RA, Lamberts SWJ, Hofland LJ. Somatostatin and dopamine receptors as targets for medical treatment of Cushing's syndrome. Rev Endocr Metab Disord. 2009;10:91102. 